\title{
Potential pitfalls related to space-based lidar remote sensing of the Earth with an emphasis on wind measurement
}

\author{
Michael J. Kavaya ${ }^{a^{*}}$, Gary D. Spiers ${ }^{b}$, Rod G. Frehlich ${ }^{\mathrm{c}}$ \\ "NASA Marshall Space Flight Center, MS SD60, Huntsville, AL 35812 USA \\ ${ }^{b}$ Univ. of Alabama in Huntsville. Center for Applied Optics, Huntsville, AL 35899 \\ ${ }^{c}$ CIRES, Campus Box 216, University of Colorado in Boulder, Boulder, CO 80309
}

\begin{abstract}
A collection of issues is discussed that are potential pitfalls, if handled incorrectly, for earth-orbiting lidar remote sensing instruments. These issues arise due to the long target ranges, high lidar-to-target relative velocities, low signal levels, use of laser scanners, and other unique aspects of using lasers in earth orbit. Consequences of misunderstanding these topics range from minor inconvenience to improper calibration to total failure. We will focus on wind measurement using coherent detection Doppler lidar, but many of the potential pitfalls apply also to noncoherent lidar wind measurement, and to measurement of parameters other than wind. Each area will be identified as to its applicability.
\end{abstract}

Keywords: Lidar, Wind. Coherent, Laser, Earth Remote Sensing

\section{INTRODUCTION}

NASA has been measuring winds with lasers since 1967, and has desired to make global tropospheric measurements with a laser radar or lidar system in space for almost as long. The primary benefits of global, accurate, high-spatial resolution wind vector profiles in the troposphere would be improved weather forecasting and validation/advancement of climate models ${ }^{\prime}$. Other U.S. government agencies and several non-US agencies also desire these measurements, either for the above listed benefits, or for specialized needs. The SPARCLE project ${ }^{2}$ was to demonstrate a coherent-detection Doppler wind lidar (CDWL) from the Space Shuttle, but was unfortunately cancelled. The first pulsed Doppler wind lidar (DWL) in space. whether on the space shuttle, space station, or a free-flyer satellite, is likely within a decade. The purpose of this paper is to discuss several issues that will need to be addressed by a DWL project/sensor team. The likelihood and impact of incorrectly handling these issues varies greatly with each issue, but it is hoped the compilation in this paper will be useful and will help a DWL development team to optimize their design for the greatest possible performance from the available hardware.

The basic geometry of the lidar remote sensing scenario is shown (not to scale) in the side and top views below. The side view shows the earth with radius $\mathrm{R}_{\mathrm{E}}$ and the satellite/lidar at altitude $\mathrm{Z}_{\mathrm{L}}$ above the earth. The lidar moves with tangential velocity $V_{L}$. We assume for now a spherical earth, circular orbit, and that the lidar beam direction lies in the plane of the orbit. Angle $\theta_{\mathrm{L}}$ is the nadir angle of the lidar beam as it leaves the orbiting instrument. This angle is a combination of the lidar hardware, its attachment to the spacecraft, and the spacecraft attitude. Angle $\theta_{\mathrm{T}}$ is the nadir angle of the lidar beam at the location of a wind measurement (the target) at altitude $\mathrm{Z}_{\mathrm{T}}$. Angle $\theta_{\mathrm{S}}$ is the nadir angle of the lidar beam as it strikes the earth's surface. The top view shows the lidar soon after crossing the equator traveling $\mathrm{S}$ to $\mathrm{N}$. Its forward direction makes a time varying angle with North (i.e., the local meridian), $\varphi_{\mathrm{N}}$. (A better rendering would show the flight path as a curving line.)

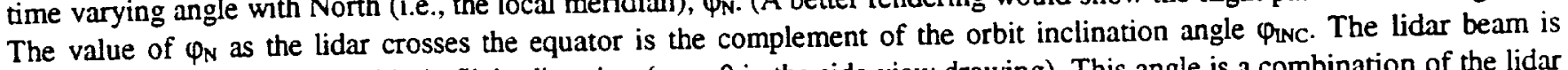
emitted at an angle $\varphi_{S}$ to the lidar's flight direction ( $\varphi_{s}=0$ in the side view drawing). This angle is a combination of the lidar hardware (primarily the scanner setting), its attachment to the spacecraft, and the spacecraft attitude. The lidar strikes its atmospheric target at latitude $\theta_{L A T}$ and altitude $Z_{T}$ where it encounters horizontal wind, $V_{H}$, having an angle $\varphi_{W}$ from North. The angle $\varphi_{N}$ may be found using

Correspondence: Email: michael.kavaya@msfc.nasa.gov; WWW: http://wwwghcc.msfc.nasa.gov/sparcle/ ; Telephone: 256 961-7803; Fax: 256-961-7772

Now with Jet Propulsion Laboratory, MS 168-214, 4800 Oak Grove Dr., Pasadena, CA 91109 


$$
\varphi_{N}=\arcsin \left[\left(\cos \varphi_{1 N C}\right) /\left(\cos \theta_{\text {LiT }}\right)\right]
$$

(Strictly. separate values of $\theta_{\text {LAT }}$ and $\varphi_{N}$ for the lidar and the wind measurement location should be calculated.) It is useful to define $R_{L E}=R_{E}+Z_{L}$, the distance of the lidar from the Earth's center. and $R_{P}=R_{L E} \sin \left(\theta_{L}\right)$. Then the nadir angle of the lidar beam at the atmospheric target is

$$
\theta_{\mathrm{T}}=\arcsin \left[R_{\mathrm{P}} /\left(\mathrm{R}_{\mathrm{E}}+\mathrm{Z}_{\mathrm{T}}\right)\right]
$$

and the distance the lidar beam travels from the lidar to the atmospheric target is found using

$$
R_{T}^{2}=\left[R_{L E}^{2}+\left(R_{E}+Z_{T}\right)^{2}-2 R_{L E}\left(R_{E}+Z_{T}\right) \cos \left(\theta_{T}-\theta_{L}\right)\right]
$$

SIDE VIEW

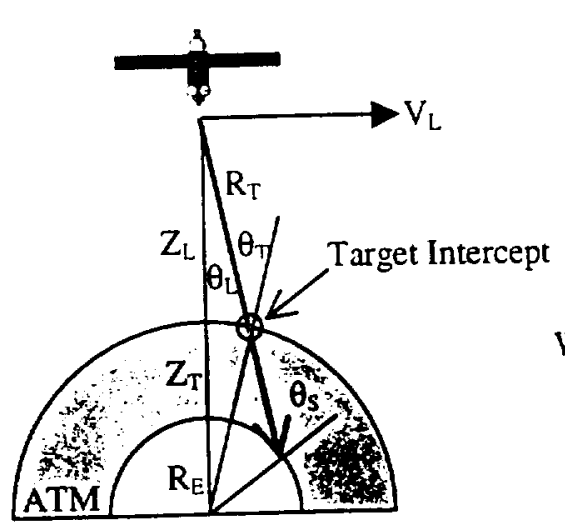

TOP VIEW

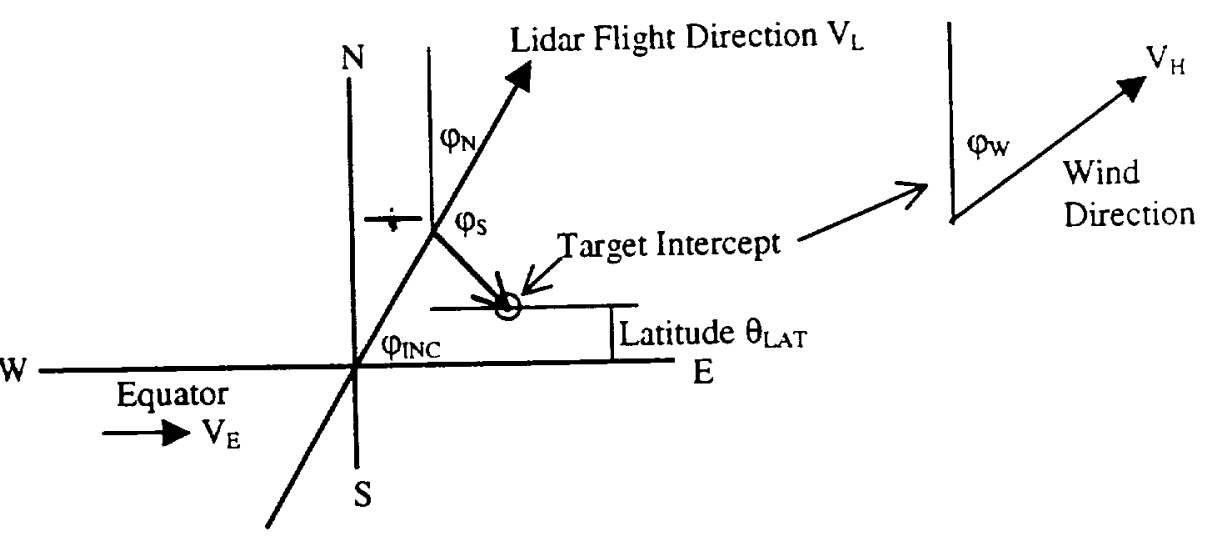

\section{ISSUES FOR LIDAR REMOTE SENSING FROM SPACE}

\subsection{Projection of line-of-sight (LOS) wind measurement into the horizontal}

Both the coherent Doppler wind lidar (CDWL) and noncoherent Doppler wind lidar (NDWL) techniques ${ }^{3-6}$ make a measurement of the wind velocity projected along the LOS of the lidar beam. The error in the wind estimate is also generated in the LOS direction. Since the wind velocity customers usually desire horizontal winds, and since comparisons of different proposed sensors/missions are frustrating if reported for different LOS directions, it is best to report wind measurement and velocity error as projected to the horizontal. Geometry reveals that an LOS wind velocity or error value should be multiplied by the inverse of the sine of the nadir angle to project it into the horizontal. But which nadir angle should be used? Since the measurement occurs at the atmospheric target, it is $\theta_{\mathrm{T}}$ that is appropriate. The above equations show that $\theta_{\mathrm{T}}$ depends on $\mathrm{Z}_{\mathrm{T}}$, the target height. So a different conversion is required for every altitude layer. As an example, let $Z_{\mathrm{L}}=833 \mathrm{~km}, \theta_{\mathrm{L}}=45 \mathrm{deg}$., and $Z_{\mathrm{T}}=3.5 \mathrm{~km}$. Then $\theta_{\mathrm{T}}=53.0 \mathrm{deg}$. and the correct multiplier to use on the LOS values is 1.25 . Mistakenly using $\theta_{\mathrm{L}}$ would yield a multiplier of 1.41 causing an error of $13 \%$. Assuming a horizontal wind of $75 \mathrm{~m} / \mathrm{s}$ parallel to the projected LOS direction, the error would be $10 \mathrm{~m} / \mathrm{s}$. The LOS velocity enor should also be multiplied by 1.25 to project it into the horizontal. For smaller lidar beam nadir angles or lower orbit heights, this multiplier becomes larger.

The angle $\theta_{\mathrm{T}}$ also depends on the radius of the earth $R_{\mathrm{E}}$. This radius varies by $21.4 \mathrm{~km}$ at different locations (not including local terrain). Thus measurement location knowledge becomes important due to velocity accuracy considerations, in order to 
look up the proper radius to use. For SPARCLE, however, the desired altitude assignment enor of the wind measurement produced a stricter requirement on knowing the local earth radius.

An elevation map of the earth is also required for velocity accuracy for a different reason. The detinition of horizontal from the wind customer's point of view is the local horizontal at the point of wind measurement. Thus the lidar's LOS wind measurement must be projected into the proper local horizontal plane. Assuming a $75 \mathrm{~m} / \mathrm{s}$ horizontal wind. the SP.ARCLE error budget required a knowledge error of local horizontal direction of approximately 5 deg.

\subsection{Removal of the spacecraft and earth rotation velocities}

Both DWLs will measure the total lidar-to-target LOS velocity. This includes the wind velocity, the earth's rotation. and the spacecraft velocity. For the calculation of the spacecraft velocity to remove trom the total measured lidar-to-target $L O S$ velocity. use the lidar beams' nadir and azimuth angles at the spacecraft. For the removal of the earth's rotational velocity. use the lidar beam's nadir and azimuth angles at the atmospheric target. Again letting $Z_{T}=833 \mathrm{~km} . \theta_{\mathrm{L}}=45$ deg.. and $Z_{\Gamma}=$ $3.5 \mathrm{~km}$ as an example, the lidar's tangential orbital velocity will be $7+42 \mathrm{~m} / \mathrm{s}$. Assuming $\varphi_{\mathrm{s}}=0$ (forward look), the correct projected LOS velocity using $\theta_{\mathrm{L}}$ is $5262 \mathrm{~m} / \mathrm{s}$. Incorrect use of $\theta_{\mathrm{T}}$ will yield a projected LOS velocity of $5943 \mathrm{~m} / \mathrm{s}$ for an error of $681 \mathrm{~m} / \mathrm{s}$.

\subsection{Knowledge error requirement of $\theta_{L}$ and $\varphi_{S}$ as a function of $\varphi_{S}$}

Since the Doppler shift due to spacecraft velocity is greatest for values of $\varphi_{S}$ near 0 and 180 deg. (fore and aft). it may seem that the required knowledge error on $\theta_{\mathrm{L}}$ and $\varphi_{\mathrm{S}}$ would be strictest at those values. This is true for $\theta_{\mathrm{L}}$. However, the rate of change of the Doppler shift vs change of $\varphi_{S}$ is greatest for values of $\varphi_{S}$ near 90 and $270 \mathrm{deg}$. (right and left). The strictest requirement on knowing the value of $\varphi_{s}$ for each lidar shot occurs at these latter values.

\subsection{Is $\mathrm{dR}_{\mathrm{T}} / \mathrm{dt}$ a velocity?}

The slant range from the lidar to the atmospheric target $R_{T}$ is continuously changing with time due to changes in the local earth radius, the spacecraft's attitude, and possibly lidar scanner motion. But although the time derivative of $R_{T}$ has units of velocity, it is not a real velocity since each individual lidar pulse generates the Doppler shifted spectrum. However, a time varying $R_{T}$ due to vertical motion of the spacecraft is a real velocity and must be removed.

\subsection{Where was the measurement made?}

Assuming a rectangular pulse of duration $\tau_{P}$ emitted at time 0 , an individual lidar pulse will propagate at speed $c$, or $-300 \mathrm{~m}$ every microsecond. At time $t$, the lead edge of the pulse will be a distance $\mathrm{ct}$ from the lidar, and the tail will be a distance $c(t$ $-\tau_{p}$ ) from the lidar. The signal recorded by the lidar's receiver is due to backscattered photons, which had to travel twice the distance from the lidar to the target that backscattered them. Therefore, the instantaneous lidar receiver signal at time $t$ is due to a slab of atmospheric backscatterers (aerosol particles and/or molecules) that is only $c \tau_{\mathrm{p}} / 2$ in thickness, even though the lidar pulse is twice as long. The end of this slab farthest from the lidar is at distance $c t / 2$. The photons at the end (tail) of the lidar pulse reach distance $\left(t-\tau_{\mathrm{P}}\right) c / 2$ and some of them are backscattered at the same time that the backscattered photons from the lead edge of the pulse return to the same distance $\left(t-\tau_{p}\right) \mathrm{c} / 2$ from their backscattered distance $\mathrm{ct} / 2$. The location of this instantaneous measurement slab moves through the atmosphere with speed $c / 2$, not the speed of light. The recorded lidar receiver signal is divided into time intervals for processing and estimating wind velocity. These intervals represent "measured" lengths of atmosphere that have a central region where the entire lidar pulse contributed to the backscartered signal, and two end regions where the fraction of the lidar pulse contributing to the signal tapers to zero.

\subsection{Do smaller optical wavelengths lead to smaller primary mirror diameters?}

There are two issues:

First. There is a danger in transferring microwave radar intuition over to lidar. The microwave radar signal power is often written as

$$
P_{R} \propto\left(\sigma D^{+}\right) /\left(R^{+} \lambda^{3}\right)
$$


Where $\sigma$ is the target cross section, $D$ is the signal antenna collection diameter. $R$ is the range to the target. and $\lambda$ is the electromagnetic wavelength ${ }^{7}$. A rule of thumb is then followed that smaller wavelengths allow smaller radar antennas. neglecting any wavelength dependence of the target cross section. This rule of thumb does not apply to remote sensing lidar. The key difference is that the radar equation assumes the target is smailer than the transmitted electromagnetic radiation. In the remote sensing lidar case. however. the atmospheric target is larger than the transmitted radiation (laser beam). The "cross section" of the lidar target is therefore the size of the lidar beam. and hence is dependent on the lidar beam's diffraction angle and the range to the target.

$$
\sigma \propto(\lambda D)^{2} R^{2}
$$

yielding

$$
P_{L} \propto D^{\prime} / R^{2}
$$

This shows that the microwave radar linkage between wavelength and receiver diameter does not apply to lidar.

Second. This pertains only to CDWL. which derives its signal from backscatter by aerosol particles in the air. The CDWL signal-to-noise ration (SNR) equation has the aerosol backscatter coefficient $\beta$ in the numerator. It is known that the aerosol backscatter coefficient generally increases as optical wavelength decreases. The rate of change of increase lies between the molecular (Rayleigh) limit of $\lambda^{4}$ and the large particle (Mie) limit of $\lambda^{0}$. This has sometimes led to the false impression that shorter wavelength lidars would have an SNR advantage that would allow smaller optical diameters to be used. But the CDWL SNR equation also has the factors hvB in the denominator, where $h$ is Planck's constant. The bandwidth B is in frequency units, $\mathrm{Hz}$. But CDWL sensors desire to measure a certain bandwidth of wind velocities. $B$ is then replaced by $B_{v} 2 / \lambda$, where $B_{v}$ is the velocity bandwidth. Since the optical frequency $v=c / \lambda$, there is actually a factor $\lambda^{2}$ in the numerator of SNR multiplying $\beta$. SNR would be independent of $\lambda$ if $\beta \propto \lambda^{-2}$. There has been a lot of research conducted to determine the wavelength dependence of $\beta$ vs. wavelength and geographic region and altitude. Chudamani et al ${ }^{8}$ participated in NASA's GLOBE aircraft flights. which gathered lidar and other data. and concluded that in the wavelength region near 1-2 microns. the dependence was $\beta \propto \lambda^{-1.9}$ at lower altitudes and $\beta \propto \lambda^{-3.0}$ at higher altitudes. Frehlich ${ }^{9}$ has extended the intermediate figure of merit SNR to a better figure of merit, the velocity estimation performance. He reported a case when comparing wavelengths of 2 and 10 microns that the velocity estimation performance became independent of wavelength if $\beta \propto \lambda^{-1.4}$.

\subsection{Is the lidar signal proportional to the receiver mirror area?}

At first glance, both DWL techniques have an SNR proportional to the square of the receiver mirror diameter. But both techniques also have other considerations. A recent paper ${ }^{10}$ discusses lidar hardware considerations for the NDWL case. The CDWL case will be discussed here. The backscattered photons, which enter the CDWL receiver, are directed onto an optical detector where they are combined with a local oscillator (LO) laser beam (heterodyne detection). The optical fields of the signal and LO photons are ideally aligned in direction, curvature, position, and polarization. Even if the lidar hardware is perfectly aligned, the long round trip time of the light from the atmosphere ( $8.4 \mathrm{~ms}$ in the previous example) may allow the attitude of the lidar to change (e.g., from spacecraft attitude changes). One component of this attitude change is predictable, namely the continuing orbit of the lidar about the center of the earth (360 deg./orbit; 1 microradian $/ \mathrm{ms}$ in our example mission). These attitude changes will cause a misalignment angle $\psi_{\text {Mis }}$ between the backscattered signal and the onboard LO optical fields. One should engineer the lidar and spacecraft for a maximum allowed value of $\psi_{\mathrm{m} I s}$, and then include the corresponding SNR loss in lidar performance predictions. Frehlich ${ }^{+}$has studied the CDWL SNR loss due to this effect as a function of the misalignment angle. For SNR loss of up to $10 \mathrm{~dB}$, a best-fit model is

$$
\begin{gathered}
\mathrm{SNR}_{\text {MIS }}=\eta_{\text {MIS }} \mathrm{SNR}_{\text {NOMIS }} \\
\eta_{\text {MIS }}=\exp \left\{-\left[\pi D \psi_{\text {MIS }} /(2.84 \lambda)\right]^{2}\right\}
\end{gathered}
$$

where $D$ is the receiver optical diameter, and $\eta_{\text {MIS }}$ is the multiplier that lowers SNR due to misaliginment. The equation reveals that larger optical diameters D will lower the SNR through this effect unless the maximum allowed misalignment

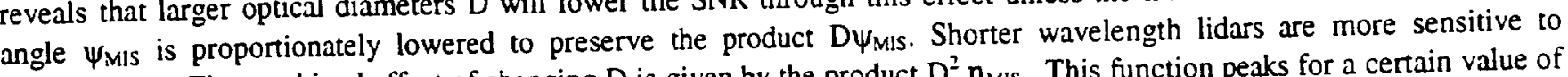
misalignment. The combined effect of changing $D$ is given by the product $D^{2} \eta_{\text {Mis }}$. This function peaks for a certain value of $D$ for a finite value of $\eta_{\text {Mis. }}{ }^{11}$ (The effect of atmospheric refractive turbulence further complicates the SNR dependence on D 
for earth-based CDWLs, but is not an issue for a CDWL in space. The effect of optical aberrations ${ }^{12}$ will also reduce SNR for smaller wavelengths.)

It was tempting at the end of Sec. 2.6 above to conclude that shorter lidar wavelength lidar will have superior velocity estimation. However. the additional effects of misalignment angle. optical aberrations, and possibly refractive turbulence prevent such a general statement.

\subsection{What is the pointing requirement?}

This question leads to confusion for a DWL mission. A better question would be "What are the pointing requirements"? A DWL mission will likely have 5 tiers of pointing requirements with 2 goals driving each: 1) to capture the signal. and 2) to obtain accurate winds. The five levels are: 1) pre-shot control of the lidar beam direction. 2) pre-shot knowledge of where the lidar is pointed. 3) pointing stability while the photons are making their round trip to the atmosphere. 4) pointing stability during the period of shot accumulation for a single LOS measurement, and 5) the tinal "as-tired" pointing knowledge of where the beam went for final velocity calculation. For SPARCLE, pointing requirements $1-4$ were involved in signal capture, and requirements +-5 were involved with final velocity accuracy.

\subsection{The optical components inside the lidar system must all be aligned much better than $\psi_{\text {MIIS }}$}

No, the requirement for misalignment angles of $\psi_{\text {MIs }}$ or less applies to the large beam (outside the lidar) side of the beamexpanding telescope. If the telescope magnification is $\mathrm{M}$, then the requirement on the small diameter side of the telescope is referenced to $\mathrm{M} \psi_{\mathrm{M} I S}$.

\subsection{Should the lidar receiver "look back" to view where the transmitted photons intercepted the atmosphere?}

This has been a controversial issue. For our example mission parameters, the spacecraft/lidar moves forward about $63 \mathrm{~m}$ while waiting for the backscattered photons to return. In order for the receiver to view the spot that the transmitter originally aimed the lidar beam at, the receiver would have to tip its view angle by approximately 35 microradians compared to the proper alignment in the absence of forward motion. (This discussion should not be confused with the tipping of the lidar due to its continuous orbit about the center of the earth. This attitude change of the lidar was discussed above, and is a separate effect that must be dealt with.) The loss of CDWL SNR vs misalignment of the signal and LO photons was discussed above. A misalignment of order 35 microradians cannot be tolerated at the SPARCLE wavelength of 2.1 microns for any reasonable value of optical diameter (a loss of $3 \mathrm{~dB}$ would occur at $D=4.5 \mathrm{~cm}$ ). Therefore the decision whether to tip the receiver axis by 35 microradians or not is crucial. A recent analysis of this problem, using the Lorentz coordinate ransformations of special relativity, has revealed that the lidar receiver should not be tipped, but rather should be aligned as if there were no translation motion ${ }^{13}$. It is helpful to take the point of view of an observer on the satellite. Consider the satellite as nonmoving, and the atmosphere as translating sideways beneath. The lidar beam is transmitted towards the atmosphere, is backscattered, and reenters the lidar receiver if the receiver is pointed in the direction of the transmitter.

\subsection{Does the choice of scanner optics affect SNR?}

Yes, different types of scanner optics may or may not reduce the effective optical diameter $D$ that should be used in the above equations. For example, a transmissive wedge scanner was to be used in the SPARCLE design to deflect the 2-micron lidar beam by 30 degrees. The wedge would then be rotated to provide a conically shaped field of regard. The outgoing beam would strike the wedge at an angle normal to one surface of the wedge. This caused the effective diameter of the output of the wedge to remain at $23.3 \mathrm{~cm}$ in one dimension, while reducing the effective diameter by $\cos \left(\theta_{\mathrm{L}}\right)$ to $20.2 \mathrm{~cm}$ in the perpendicular direction. This effect may or may not lower SNR depending on the misalignment angle effect discussed above. An alternate option would be to use the wedge in a symmetric optical arrangement where optical wavefronts on both sides of the wedge strike the wedge at an angle of $15 \mathrm{deg}$. from normal incidence. There would then be no diameter reduction in one dimension, but the wedge would be larger and heavier for an equal beam diameter, and the volume required to allow the wedge to rotate would be larger. 

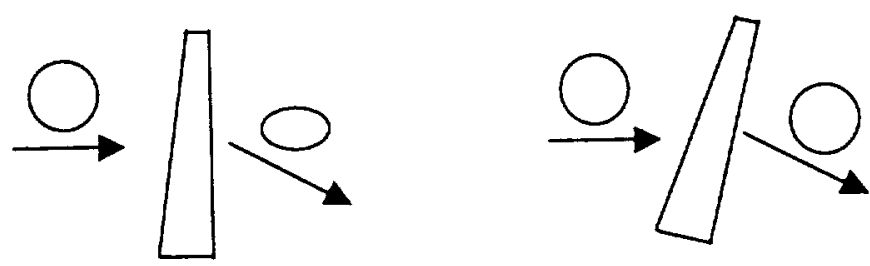

2.12 As SNR decreases, does the wind velocity error increase?

This is true for NDWL. which calculates the wind velocity from the results of several intensity measurements of the signal photons. However. CDWL is fundamentally different in that no intensity measurements are needed for velocity estimation. The optical detector is used as a mixer, along with the LO beam, to translate a perfectly preserved version of the optical signal's spectrum to much lower (e.g., MHz) frequencies. Then, either electronic components, or a digitizer and computer. are used to estimate the velocity. This estimation may be simplistically pictured as taking a Fourier transform of a finite time interval of the signal data, and operating on its frequency spectrum to find the frequency bin with the largest value. This is a random process. When the largest value frequency bin corresponds to the correct wind velocity, the estimate is "good" and is very accurate. When noise causes a frequency bin to have a larger signal than the true wind velocity, the estimate will be "bad" and will be uniformly distributed over the total range of frequencies of the Fourier transform (assuming the desired tlat noise floor). The net effect of this fundamental difference is that the CDWL velocity estimate will be very accurate whenever the estimate is "good". As SNR decreases, the main effect will be an increase in the percent of estimates that are "bad". The "good" estimates will remain accurate, but less likely for lower SNR. When the aerosol reflectance or quantity in a certain volume of the atmosphere is small, the CDWL will have lower SNR.

For this reason, NDWL must address the issue of sufficient velocity accuracy, while CDWL must address the issue of sufficient coverage of the atmosphere.

The heterodyne detection (or spectral domain or tone detection) DWL technique is extremely efficient with photons. A recent paper by Johnson and Townes ${ }^{14}$ states "heterodyne detection can allow measurement of the phase of a single-frequency wave to a precision limited only by the uncertainty principle".

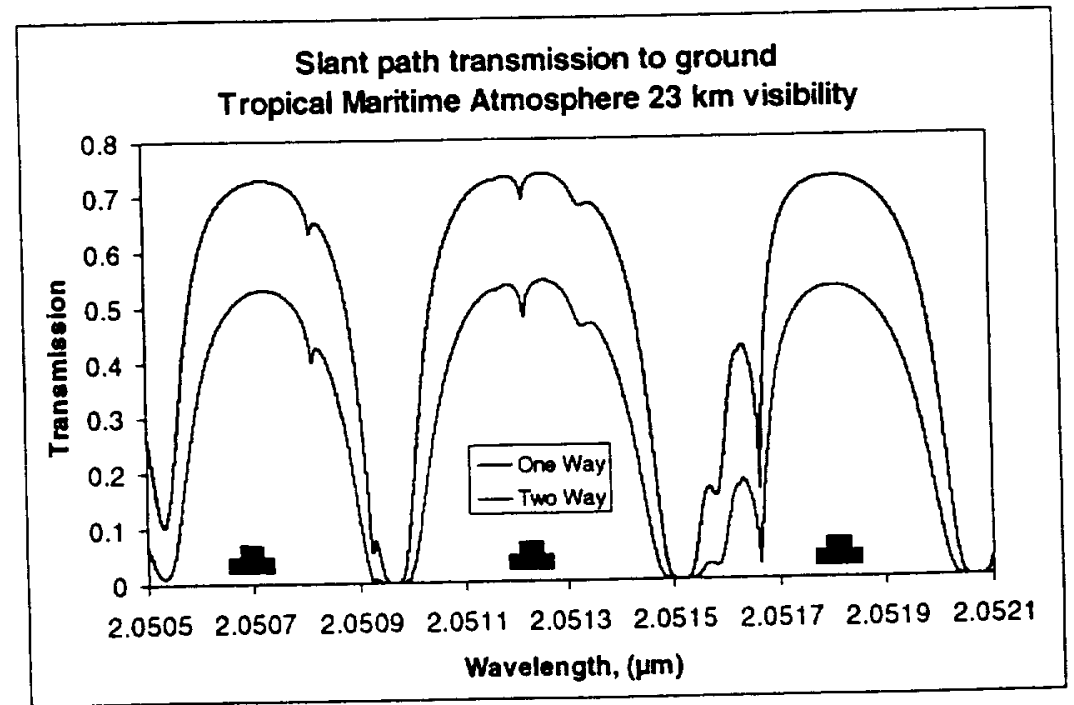

\subsection{The absolute laser wavelength is unimportant}

If there were no atmospheric extinction of the lidar beam power, we could allow the transmitted laser wavelength to vary considerably from shot to shot, simply recording the transmitter to LO frequency difference for each shot for later velocity calculation. But the atmospheric extinction varies dramatically with wavelength and must be taken into consideration. The 
laser wavelength must be chosen and controlled to remain in an atmospheric "window" of high transmission. The figure above shows the 1-way and 2-way (round trip) transmission of the atmosphere for a wind measurement at ground level for the SPARCLE mission parameters of $Z_{L}=300 \mathrm{~km}$ and $\theta_{\mathrm{L}}=30 \mathrm{deg}$. (The atmospheric transmission is better for higher altitude wind measurements.) The SPARCLE wavelength of 2.0518 microns is inside the right-hand "window" of the 3 shown. The abscissa scale of the figure has tick marks separated by $0.2 \mathrm{~nm}$. The nominal SPARCLE lidar pulse spectral width of $2.5 \mathrm{MHz}$ FWHM would correspond to only $0.00003+\mathrm{nm}$, and easily fits within the "windows". But recall that the SPARCLE scanner was to aim the lidar pulse at different azimuth angles. This causes an azimuth dependent Doppler shift due to the large relative velocity of the lidar and the atmospheric target. Not including various margins. e.g., for spacecraft attitude variations. the extreme (fore and aft azimuths) Doppler shifts expected for the SPARCLE mission were $\pm 3770 \mathrm{MHz}$. or $7540 \mathrm{MHz}$ neglecting platform attitude variations. This corresponds to about $0.1 \mathrm{~nm}$. and the full range of shift is shown by the lower horizontal bars in the figure. Depending on the width of the atmospheric "window", and how centered the laser wavelength is in the "window", the SNR may depend on azimuth angle, which is undesirable. However, we derived the frequency shift from the velocity changes using the lidar conversion factor of $2 / \lambda$. This is accurate for the Doppler shiti seen by the lidar receiver. The factor of 2 in the conversion factor arises from the fact that both the source of the lidar light and the lidar receiver are moving with respect to the target. The atmosphere, conversely, is unaware of the eventual detection of the backscattered light by a moving receiver, and the appropriate conversion factor for it is $1 / \lambda$. The shorter upper horizontal bars in the figure are the correct extent of the Doppler shift between the fore and aft scanner positions for atmospheric transmission considerations.

\subsection{Tuning out the large Doppler shift}

Rather than have a detector and receiver chain that can handle the $7.5 \mathrm{GHz}$ range of Doppler shifts due to the scanner's azimuth angle, the SPARCLE instrument planned to tune out most of this shift with a tunable laser ${ }^{15}$. This is straightforward if the laser that is tuned is the LO laser. But for technology reasons, SPARCLE planned to tune its transmitter laser and keep the LO laser frequency constant. This would have necessitated an azimuth dependent conversion factor between frequency and velocity since the transmitter frequency would be varying.

\subsection{A large nadir angle $\theta_{L}$ is important to have good coverage of the earth}

It's true that larger nadir angles $\theta_{\mathrm{L}}$ will have a larger cross-track "field of regard" swath width as the scanner turns. But the current technology status of both CDWL and NDWL techniques require multiple shot accumulation (combination) in order to make each individual LOS wind measurement. This forces the DWL to stop its scanner, to stare in one direction, and to draw "straight lines" (picture them on the earth's surface) for a single LOS measurement. The length of these lines depends on the required number of accumulated shots, the pulse repetition rate $f_{L}$ of the laser, and the forward speed of the spacecraft (adjusted to the wind measurement altitude). Assuming that the along-track desired horizontal resolution of the wind velocity measurement is $100 \mathrm{~km}$, and assuming the $833-\mathrm{km}$ example mission parameters used above, the wind measurement location moves forward by $100 \mathrm{~km}$ in about $15 \mathrm{~s}$. Making up numbers for this example, let $\mathrm{f}_{\mathrm{L}}=12 \mathrm{~Hz}$, and $\mathrm{N}_{\mathrm{A}}=45$ be the number of accumulated shots needed per LOS wind measurement. Each LOS wind measurement requires $3.75 \mathrm{~s}$ of lidar "staring" in the same perspective. Not counting time spent in moving the scanner to a new azimuth setting, a maximum of 4 "straight line" LOS wind measurements are possible at the $100 \mathrm{~km}$ resolution, if the measurement must be repeated for every $100 \mathrm{~km}$ of forward travel with no gaps. For vector wind measurements, fore and aft "straight line" LOS measurements should be paired. So, simplistically, 2 vector wind measurements can be spaced as desired in the cross-track direction within the field of regard of the scanner.

The lidar situation is vastly different than passive imaging remote sensing, for which swath width or coverage is a key parameter. The measurement lines in the along-track direction are very narrow in the cross-track direction. For CDWL they are on the order of $10 \mathrm{~m}$ wide. In our example, the lidar beam strikes the atmospheric target about $900 \mathrm{~km}$ from the point directly below the satellite. On the following orbit 101 minutes later, the earth will have rotated $2800 \mathrm{~km}$ at the equation with respect to the lidar. This distance tapers to 0 at the poles. It is not obvious in the DWL case how science value will increase as the field of regard is increased. But it is clear that the larger range to the target and decreased atmospheric transmission from larger nadir angles will lower the DWL SNR.

Since the DWL cannot provide total blanket coverage in the cross-track direction, perhaps blanket coverage in the alongtrack direction is not needed. For example, a wind measurement in one $100-\mathrm{km}$ length of spacecraft forward motion could be followed by 1 or more $100-\mathrm{km}$ lengths with no wind measurement (no lidar shots). This would allow twice the number of 
lidar shots to be directed into the measured $100-\mathrm{km}$ length if the along-track duty cycle were $50 \%$, three times the shots if the along-track duty cycle were $33 \%$, etc. This trading of along-track measurement duty cycle for better vertical coverage in the CDWL case, and for better wind accuracy in the NDWL case, can only be taken as far as the orbit geometry permits. The extra lidar shots can only be directed into the desired $100-\mathrm{km}$ length if the reach of the scanner allows it. Higher orbit heights and larger nadir angles allow greater flexibility in making this trade-off.

\subsection{What happens near the 1-photon limit?}

The CDWL wind measurement theory (and experimental contirmation ${ }^{16}$ ) reveals that excellent performance is obtained with a very small number of photons. The question arises whether the behavior will change as the 1-photon limit is reached. Several excellent papers have addressed this question, from both semi classical and quantum mechanical viewpoints. and concluded that there is no effect ${ }^{17-19}$.

\subsection{The receiver must have an analog to digital converter (ADC) with a large dynamic range}

A requirement for a large receiver dynamic range due to the great variation of the target reflectance (aerosols, molecules. clouds, land, water, ice) is appropriate for NDWL where the detected signal is proportional to the intensity of the backscattered field. However, CDWL uses an LO laser and heterodyne detection. This technique produces a signal power out of the optical detector that is proportional to the square root of the intensity of the backscattered field ${ }^{20}$. Secondiy. the heterodyne detection technique is arranged to be shot noise limited by using an LO power much larger than the signal power. Changes in the signal power have minimal effect on the total power. Thirdly, the primary measurement is of the frequency of the signal, and not its intensity. These combined considerations allow an analog-to-digital converter (ADC) with less dynamic range to be satisfactory. There remains a need for a rigorous study of the wind measurement performance as a function of receiver dynamic range. 


\section{ISSUE APPLICABILITY TABLE}

\begin{tabular}{|c|c|c|c|}
\hline & $\begin{array}{l}\text { Coherent Doppler Wind } \\
\text { Lidar }\end{array}$ & $\begin{array}{l}\text { Noncoherent Doppler Wind } \\
\text { Lidar }\end{array}$ & $\begin{array}{c}\text { Non-wind Lidar Remote } \\
\text { Sensing }\end{array}$ \\
\hline $\begin{array}{l}\text { Projection of line-of-sight } \\
\text { (LOS) wind measurement } \\
\text { into the horizontal }\end{array}$ & $\checkmark$ & ${ }_{2}$ & \\
\hline $\begin{array}{l}\text { Removal of the spacecraft } \\
\text { and earth rotation velocities }\end{array}$ & $\checkmark$ & $\boldsymbol{V}$ & \\
\hline $\begin{array}{l}\text { Knowledge error } \\
\text { requirement of } \theta_{L} \text { and } \varphi_{s} \text { as a } \\
\text { function of } \varphi_{S}\end{array}$ & $\checkmark$ & $\checkmark$ & \\
\hline Is $\mathrm{dR}_{\mathrm{l}} / \mathrm{dt}$ a velocity? & $\checkmark$ & $\checkmark$ & \\
\hline $\begin{array}{l}\text { Where was the measurement } \\
\text { made? }\end{array}$ & $\checkmark$ & $\checkmark$ & $\checkmark$ \\
\hline $\begin{array}{l}\text { Do smaller optical } \\
\text { wavelengths lead to smaller } \\
\text { primary mirror diameters? }\end{array}$ & $\checkmark$ & & \\
\hline $\begin{array}{c}\text { Is the lidar signal } \\
\text { proportional to the receiver } \\
\text { mirror area? }\end{array}$ & $V$ & $\begin{array}{l}\text { Not sure. Need relationship } \\
\text { of SNR and misalignment } \\
\text { angle and mirror diameter }\end{array}$ & \\
\hline $\begin{array}{l}\text { What is the pointing } \\
\text { requirement? }\end{array}$ & $\checkmark$ & 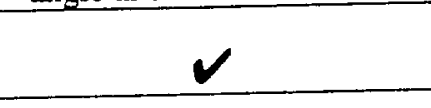 & \\
\hline $\begin{array}{l}\text { The optical components } \\
\text { inside the lidar system must } \\
\text { all be aligned much better } \\
\text { than } \psi_{\text {MIS }}\end{array}$ & $V$ & & \\
\hline $\begin{array}{l}\text { Should the lidar receiver } \\
\text { "look back" to view where } \\
\text { the transmitted photons } \\
\text { intercepted the atmosphere? }\end{array}$ & $\checkmark$ & $\checkmark$ & $\checkmark$ \\
\hline $\begin{array}{c}\text { Does the choice of scanner } \\
\text { optics affect SNR? }\end{array}$ & $\checkmark$ & $\checkmark$ & $\checkmark$ \\
\hline $\begin{array}{l}\text { As SNR decreases, does the } \\
\text { wind velocity error increase? }\end{array}$ & $\checkmark$ & & \\
\hline $\begin{array}{l}\text { The absolute laser } \\
\text { wavelength is unimportant }\end{array}$ & $V$ & $\checkmark$ & $\checkmark$ \\
\hline $\begin{array}{c}\text { Tuning out the large Doppler } \\
\text { shift }\end{array}$ & $V$ & & \\
\hline $\begin{array}{l}\text { A large nadir angle } \theta_{\mathrm{L}} \text { is } \\
\text { important to have large } \\
\text { measurement coverage of the } \\
\text { earth }\end{array}$ & $V$ & $\checkmark$ & $V$ \\
\hline $\begin{array}{l}\text { What happens near the l- } \\
\text { photon limit? }\end{array}$ & $\mathscr{\nu}$ & & \\
\hline $\begin{array}{c}\text { The receiver must have an } \\
\text { analog to digital converter } \\
\text { (ADC) with a large dynamic } \\
\text { range }\end{array}$ & $\checkmark$ & & \\
\hline
\end{tabular}




\section{ACKNOWLEDGMENTS}

The authors greatly appreciate the funding provided by Dr. Ramesh K. Kakar at NASA Headquarters Earth Science Enterprise. and by Dr. Stephen A. Mango at the National Polar-orbiting Operational Environmental Satellite System (NPOESS) Integrated Program Otfice (IPO). They also acknowledge many helpful discussions with Farzin Amzajerdian. David Emmitt. Sammy Henderson. and Tim Miller.

\section{REFERENCES}

1. W. A. Baker. G. D. Emmitt. F. Robertson. R. A. Atlas, J. E. Molinari, D. A. Bowdle. J. Paegle, R. M. Hardesty. R. T Menzies. T. N. Krishnamurti. R. A. Brown, M. J. Post, J. R. Anderson. A. C. Lorenc. and J. McElroy, "Lidar-Measured Winds from Space: A Key Component for Weather and Climate Prediction." Bull. American Meteorological Society 76(6), 869-888 (1995)

2. M. J. Kavaya and G. D. Emmitt. "The Space Readiness Coherent Lidar Experiment (SPARCLE) Space Shuttle Mission." Proc. SPIE 3380, 2-11, (1+ April 1998)

3. R. T. Menzies, "Doppler lidar atmospheric wind sensors: a comparative performance evaluation for global measurement applications from earth orbit," Appl. Opt. 25, 2546-2552 (1986)

4. R. G. Frehlich. "Coherent Doppler lidar measurements of winds," in "Trends in Optics: Research, Developments and Applications," ed. by Anna Consortini, Academic Press (1996)

5. M. J. McGill and J. D. Spinhirne, "Comparison of two direct-detection Doppler lidar techniques," Opt. Eng. 37, 2675 2686 (1998)

6. J. A. McKay. "Comparing the Intrinsic, Photon Shot Noise Limited Sensitivity of Coherent and Direct Detection Doppler Wind Lidar," Digest of the 10th Biennial Coherent Laser Radar Technology and Applications Conference. 106109. Mount Hood, OR (28 June - 2 July 1999); also NASAJCP-1999-209758 (Nov. 1999)

7. R. J. Doviak and D. S. Zrnic, "Doppler Radar And Weather Observations," Sec. 3.4, Academic Press (1993)

8. S. Chudamani. J. D. Spinhirne, and A. D. Clarke, "Lidar aerosol backscatter cross sections in the 2-um near-infrared wavelength region," Appl. Opt. 35, $4812-4819$ (1996)

9. R. Frehlich, "Comparison of 2- and 10- $\mu \mathrm{m}$ Coherent Doppler Lidar Performance," J. Atmospheric and Oceanic Tech. 12. 415-420 (1995)

10. J. A. McKay, "Fabry-Perot etalon aperture requirements for direct detection Doppler wind lidar from Earth orbit," Appl. Opt. 38, 5859-5866 (1999)

11. M. J. Kavaya, G.D. Spiers, E.S. Lobl, J. Rothermel, and V.W. Keller, "Direct global measurements of tropospheric winds employing a simplified coherent laser radar using fully scalable technology and technique," Proc. SPIE Vol. $221+$. 237-249, (1994)

12. G.D. Spiers, "The effect of optical aberrations on the performance of a coherent Doppler lidar", Tenth Biennial Coherent Laser Radar Technology and Applications Conference Digest, Mt. Hood, Oregon, June 28 - July 2, 170 - 173, (1999); also NASACP-1999-209758 (Nov. 1999)

13. V. S. R. Gudimetla and M. J. Kavaya, "Special Relativity Corrections for Space-Based Lidars," Appl. Opt. 38(30), 637t6382 (1999)

14. M. A. Johnson and C. H. Townes, "Quantum effects and optimization of heterodyne detection," Optics Communications $179,183-187$ (2000)

15. G.D. Spiers, F. Amzajerdian, S.C. Johnson, "Design considerations for a receiver for a space based coherent Doppler lidar operating in the two micron wavelength region", OSA 1995 Technical Digest Series Volume 19 "Coherent Laser Radar", 58 - 61, (1995)

16. R. Frehlich, S. M. Hannon, and S. W. Henderson, "Coherent Doppler lidar measurements of winds in the weak signal regime," Applied Optics 36, 3491-3499, (1997)

17. L. Mandel and E. Wolf, "Photon Statistics and Classical Fields," Phys. Rev. 149, 1033-1037 (1966)

18. J. H. Shapiro, "Quantum Noise and Excess Noise in Optical Homodyne and Heterodyne Receivers," IEEE J. Quantum Electronics QE-21, 237-250 (1985)

19. P. J. Winzer and W. R. Leeb, "Coherent lidar at low signal powers: basic considerations on optical heterodyning," J. Modern Optics 45, 1549-1555 (1998)

20. R. G. Frehlich and M. J. Kavaya, "Coherent laser radar performance for general atmospheric refractive turbulence," Applied Optics 30, 5325-5352 (1991)

21. There are steps to having a very successful life. First, don't tell people everything you know. 